\title{
Realising the potential of health information technology to enhance medication safety
}

\author{
Aziz Sheikh
}

Centre for Medical Informatics, Usher Institute, University of Edinburgh, Edinburgh, UK

\section{Correspondence to} Professor Aziz Sheikh, Centre for Medical Informatics, Usher Institute, University of Edinburgh, Edinburgh EH8 9DX, UK; aziz.sheikh@ed.ac.uk

Accepted 6 September 2019 Published Online First 13 September 2019

\section{SLinked}

- http://dx.doi.org/10.1136/ bmjqs-2019-009609

\section{Check for updates}

(c) Author(s) (or their employer(s)) 2020. No commercial re-use. See rights and permissions. Published by BMJ.

To cite: Sheikh A.

BMJ Qual Saf 2020;29:7-9.
There is now widespread awareness of the very considerable burden of harm and associated costs resulting from medication errors, which, in turn, has stimulated national and international drives to reduce medication-associated harm. In parallel, there is a growing appreciation that health information technology (HIT) has the potential to reduce the risk of medication errors. There is, however, a wide gulf between HIT as a structural intervention and its translation into improvements in care processes, and a wider gulf still between the process of care and improvements in health outcomes. ${ }^{1}$ What matters to patients, and their loved ones, is of course avoidance of actual harm and it is for this reason that the WHO, in launching its Third Global Safety Challenge, called it 'Medication Without Harm'.2

Governments across the world are investing substantial sums of money in moving care from paper-based records to electronic health record (EHR) infrastructures. A key driver for this move is the belief that this will result in substantial improvements in patient safety. ${ }^{3} \mathrm{~A}$ high frequency of medication errors and preventable adverse drug events have been documented in many studies of patient safety problems, making medication safety an obvious place to start. Yet, the analysis by Holmgren et al reported in this edition demonstrates that current EHRs would fail to prevent over onethird of potentially serious medication errors in a sample of 1527 hospitals in the USA. ${ }^{4}$ A key strength of their study was that they were able to track progress in preventing such medication errors over a 7-year period (ie, from 2009 to 2016). Despite this coinciding with a period in which the largest national investments in EHRs were made, through the US Health
Information Technology for Economic and Clinical Health Act, ${ }^{5}$ there were only relatively modest improvements in hospital safety performance when judged using a standardised (and largely unchanged) set of 44 simulated medication orders.

It is important to try to understand why there were such modest effects in the translation from structural intervention to the process of ordering medications. The reasons are likely to be multifaceted, including the fact that much of the strongest evidence on EHRs improving medication safety comes from clinician enthusiast-led, 'home-grown' and extensively customised EHRs that had improvements in medication safety as a core goal. ${ }^{6}$ In contrast, health systems across the world are largely now implementing commercial 'off-the-shelf' EHRs, which were primarily designed to support administrative tasks related to billing and documentation rather than to improve patient safety. Furthermore, the defensive stance adopted by these commercial EHR vendors has resulted in what many clinicians consider inappropriate alerts leading to the widely recognised phenomenon of alert fatigue and the inadvertent over-riding of clinically important warnings. ${ }^{7}$ More substantially still, it is naïve to consider that computerised provider order entry (CPOE) or computerised decision support systems (CDSS) alone would be able to prevent all or even the majority of medication errors. Rather, what is needed is for HIT interventions to be framed within a broader set of approaches that pay due consideration to the sociotechnical factors that are important to determining how HIT is deployed and implemented in practice. ${ }^{8}$

Looking ahead, I believe there are six key issues that need to be addressed to 
realise the potential of HIT to improve medication safety. I consider these, in turn below.

First, there is a need for more accurate data on the absolute and relative burden of preventable harm associated with different medications across care settings - that is, both in hospital and community care contexts-and an appreciation of which subpopulations are at highest risk of these errors. Such information is crucial to build a common understanding of the medications, patients and clinical settings that are most amenable to HIT-based interventions and on which we therefore ought to focus our efforts. ${ }^{9}$

Second, and related to this, there is a need to develop valid and reliable indicators of errors associated with these drugs to allow meaningful benchmarking and an assessment of progress in enhancing patient safety over time or in relation to the effectiveness of interventions. Some progress has been made in this respect through the work of the Leapfrog Group in the USA. In fact, the study reported by Holmgren and colleagues ${ }^{4}$ involves a CPOE assessment test designed by investigators at Brigham and Women's Hospital in Boston and the University of Utah, but used by the Leapfrog Group in their Annual Safe Practices Survey, distributed nationally to US hospitals with results publicly reported. In the UK, a limited number of medication safety indicators have been developed for the drugs that are most commonly implicated in serious potentially avoidable harm in hospital settings ${ }^{10}$ and, importantly, also in primary care. $^{11}$

Third, there is a need to develop interventions that capitalise on the increasingly ubiquitous EHR infrastructures and accompanying CPOE and CDSS functionality, but which also attend to wider sociotechnical considerations. Such complex interventions will invariably need to include one or more of additional educational, training or behavioural change dimensions and are likely to include feedback loops if we are to use these technologies to achieve sustained change.

Fourth, there is then a need to evaluate the acceptability, effectiveness and cost-effectiveness of these complex HIT-based interventions using robust study designs, preferably randomised controlled trials. The choice of outcomes in such trials is crucially important. ${ }^{12}$ Wherever possible, clinical endpoints are preferred over process measures. ${ }^{13}$ For those interventions found to be effective, there is then the need to draw on implementation science to find ways of embedding the innovation into routine clinical care at scale.

Fifth, we need to develop mechanisms to enable standardised reporting of progress in enhancing medication safety nationally. The WHO co-led annual Global Ministerial Summits on Patient Safety now provide a high-profile forum for presenting updates on progress and for the sharing of expertise, experiences and lessons learnt.
Finally, we need far more open in reporting on the (many) limitations of EHRs in hampering progress towards the goal of medication safety together with mandatory reporting of any new HIT/EHR-associated risks to medication safety. Such transparency should lead to more informed choices about the relative strengths and limitations of different EHRs and, more importantly, provide important insights into how to improve the design of EHRs.

The WHO'S Third Global Safety Challenge, the recent World Health Assembly Resolution on Patient Safety ${ }^{14}$ and the continuing policy interest in EHRs have combined to provide an unparalleled opportunity to realise the potential of HIT to enhance medication safety. We owe it to our patients to work in a collaborative, coordinated way to achieve the much-needed HIT-enabled leap forward towards the goal of Medication Without Harm.

Acknowledgements I gratefully acknowledge support from NIHR Programme Grants for Applied Research, the Policy Research Programme of the Department of Health and Social Care and Health Data Research UK.

Funding The authors have not declared a specific grant for this research from any funding agency in the public, commercial or not-for-profit sectors.

Competing interests None declared.

Patient consent for publication Not required.

Provenance and peer review Commissioned; internally peer reviewed.

\section{REFERENCES}

1. Donabedian A. Evaluating the quality of medical care. Milbank Mem Fund Q 1966;44:166-206.

2. Donaldson LJ, Kelley ET, Dhingra-Kumar N, et al. Medication without harm: who's third global patient safety challenge. The Lancet 2017;389:1680-1.

3. Cresswell K, Bates DW, Sheikh A. Six ways for governments to get value from health it. The Lancet 2016;387:2074-5.

4 Holmgren AJ, Co Z, Newmark L, et al. Assessing the safety of electronic health records: a national longitudinal study of medication-related decision support. BMJ Qual Saf 2020;29:52-9

5. Washington V, DeSalvo K, Mostashari F, et al. The HITECH era and the path forward. N Engl J Med Overseas Ed 2017;377:904-6.

6. Chaudhry B, Wang J, Wu S, et al. Systematic review: impact of health information technology on quality, efficiency, and costs of medical care. Ann Intern Med 2006;144:742-52.

7. Kesselheim AS, Cresswell K, Phansalkar S, et al. Clinical Decision Support Systems Could Be Modified To Reduce 'Alert Fatigue' While Still Minimizing The Risk Of Litigation. Health Aff 2011;30:2310-7.10.1377/hlthaff.2010.1111

8. Black AD, Car J, Pagliari C, et al. The impact of eHealth on the quality and safety of health care: a systematic overview. PLoS Med 2011;8:e1000387.10.1371/journal.pmed.1000387

9. Sheikh A, Rudan I, Cresswell K, et al. Agreeing on global research priorities for medication safety: an international prioritisation exercise. J Glob Health 2019;9:010422.

10. Thomas SK, McDowell SE, Hodson J, et al. Developing consensus on hospital prescribing indicators of potential 
harms amenable to decision support. Br J Clin Pharmacol 2013;76:797-809.

11. Howard RL, Avery AJ, Slavenburg S, et al. Which drugs cause preventable admissions to hospital? A systematic review. $\mathrm{Br} \mathrm{J}$ Clin Pharmacol 2007;63:136-47.

12. Avery AJ, Rodgers S, Cantrill JA, et al. A pharmacistled information technology intervention for medication errors (pincer): a multicentre, cluster randomised, controlled trial and cost-effectiveness analysis. The Lancet 2012;379:1310-9.

13. Dreischulte T, Donnan P, Grant A, et al. Safer prescribing - a trial of education, informatics, and financial incentives. N Engl J Med Overseas Ed 2016;374:1053-64.

14. Who. patient safety. A72/26, 25 March, 2019. Available: https://apps.who.int/gb/ebwha/pdf_files/WHA72/A72_26-en. pdf [Accessed 28 Aug 2019]. 